\title{
The Research on Inducible Strategy to Optimize the Staff Configuration
}

\author{
Weiwei Sha \\ Faculty of Information Engineering and Automation \\ Kunming University of Science and Technology \\ Kunming, China \\ e-mail: shaww88@163.com \\ Jingru Su \\ Faculty of Information Engineering and Automation \\ Kunming University of Science and Technology \\ Kunming, China \\ e-mail: 18811505312@163.com
}

\author{
Zengli Liu \\ Faculty of Information Engineering and Automation \\ Kunming University of Science and Technology \\ Kunming, China \\ e-mail: liuzengli@ hotmail.com \\ Bing Chen \\ Faculty of Information Engineering and Automation \\ Kunming University of Science and Technology \\ Kunming, China \\ e-mail: gdstucb@sina.com
}

\begin{abstract}
In this paper, production-oriented enterprises are used as the research objects. Based on the forward master-slave framework of two-tier decision model, superiors affect even intervene production decisions of subordinate departments, this must be taken into consideration. That is to say, in the inducible decision-making mechanism strategy of two-tier model, optimization algorithm for the allocation about sharing staff and its own staff is put forward, its aim is to achieve maximum NPV (Net Present Value).
\end{abstract}

Keywords: inducible strategy; two-tier model; optimization algorithm.

\section{INTRODUCTION}

Multiple projects are encompassed by the production-oriented enterprises, but the staff is limited. In order to maximize the profitability, enterprises must consider all projects, also consider appropriate allocation of personnel across multiple projects and wealthy differences simultaneously. Hierarchical personnel assignment of multi-project coexistence is a complex management issues. The forward master-slave framework of two-tier decision model [1] may be used to describe the problem.

\section{REQUIREMENTS ANALYSIS OF TWO-INDUCIBLE MODEL}

There is often limited staff allocation between the multiple subordinate units in the production and construction industry. From the forward master-slave decision-making mechanisms, which problem we frequently encountered about staff assignments is how higher-level units do allocation with limited personnel between sectors, so that the departments and themselves can achieve the goal to the optimal production. Meanwhile various departments organize production in accordance with personnel superiors assigned to make their own goals optimally. However, in the real decision-making, there are certain advantages superior

Impact even interfere with subordinate departments on production decisions. This also reflects the higher leadership. Such personnel assignment problems can be described as an inducible decision-making mechanism of the two-tier strategy [2].

In the inducible strategy, main task of superior is how to affect subordinates decision-making, which give superiors a favor conversely. Between the upper and lower levels should adhere to the following guidelines. First, before decision-making, superiors should announce the chosen strategy. Second, in the decision-making process, the higher must act according to the announced policy. The last, subordinates only act according to their own optimization, no other considerations. The above three criteria indicate that superiors can't deceive subordinates. Also, superiors can't change policy after the decisions taken by subordinates. Subordinates will not take threatening means to force superiors to change strategy.

\section{Model Two-InduciBle Decisions Assume THAT OWN RESOURCES ARE NOT LIMITED}

In the production process, not only the allocation of shared personnel will affect the achievements about superiors and production departments, changing its own personnel will also affect the goal. This article discusses the case of its own personnel are not limited.

Suppose headquarters possess $m$ kind of staff to be assigned to $\mathrm{p}$ branch subordinate. Each branch of the production department should use $\mathrm{m}$ - kind of shared personnel and own staff to arrange project tasks. Each branch subordinate has its own staff, to simplify the problem, the allocation of its own staff of each branch is not considered. Assumed that the number of personnel of each branch subordinate can meet the required project activities using its own staff, and activity duration is unchanged. The activity duration using shared personnel is determined by the number of personnel assigned to the branch. Suppose activity duration of shared member is inversely proportional to the amount of allocation, then the activity duration is equal to the amount of active workload divided by shared staff. Headquarters aim to the goal that each branch fulfill 
tasks in a predetermined period and use the smallest amount of shared staff. The lower each branch target the maximum NPV. In addition, if the subordinate decision does not comply with the requirements of higher expectations, headquarters has the ability to influence lower, so that subordinates are induced to conform to the expectations of the headquarters. The following mathematical model which describe the personnel allocation among multiple projects.

$$
\begin{aligned}
& \min F(R)=\varepsilon \sum_{L=1}^{m} \sum_{k=1}^{P} R_{k L}+\sum_{k=1}^{P}\left(\max \left(\omega_{k}^{*}-\omega_{k}, 0\right)\right) \\
& \text { s.t. }\left\{\begin{array}{c}
\sum_{k=1}^{P} R_{k L}=Q_{L}, \quad L=1, \ldots, m \\
R_{k L} \geq 0, \quad k=1, \ldots, p
\end{array}\right.
\end{aligned}
$$

Here $\omega_{k}$ is the underlying goal of the k-the policy-makers objective function. $\omega^{*} \mathrm{k}$ refers to the k-the branch prediction value of gross economic value. $R_{k L}$ refers to the number of shared personnel assigned to the $\mathrm{k}$-the branch of the L-species.

Eq. (1) is the objective function for the upper decision. The first is a secondary objective. $\varepsilon$ is an arbitrarily small positive number(as $10^{-4}$ ). The second term in this equation represents the tardiness which refers to in each project the production cycle relative to the specified completion date. The reason it added to the objective function is that the lower is to maximize NPV. Considering such a situation, when using shared personnel to assign activities, one or several are not in the critical path, the allocation will not affect the total economic value together with personnel changes within a certain range, so there is no unique solution. To overcome this situation, the upper objective function adds ancillary target which requires personnel consumers are minimal.

If starting from the perspective of the headquarters planning layer, equation (2) constitutes the constraints of decision problem. The problem to be solved is the total amount of personnel under certain circumstances, and how to assign shared personnel to each subordinate production department so that can achieve the desired economic value, and the total personnel consumption is minimal. Eq. (3) and (4) depict the problems the lower k-the factory may encounter. It needs to solve problems how to organize their own production according to the amount of people assigned by the top decision-makers, and its goal is to make their own economic value as large as possible. $Q_{L}$ refers to the number of the L-the shared personnel; ${ }^{c}{ }{ }$ refers to the unit-value of i-the products of k-the branch; ${ }^{x_{i k}}$ refers to the i-the products of $\mathrm{k}$-the branch; $d_{i k L}$ refers to the consumption of the $\mathrm{k}$-the branch of $\mathrm{i}$-the products $\mathrm{L}-$ the share personnel.

$$
\begin{gathered}
\max \omega_{k}=\sum_{i=1}^{n} c_{i k} x_{i k}, \quad k=1, \ldots, p ; i=1, \ldots, n \\
\text { s.t. }\left\{\begin{array}{c}
\sum_{k=1}^{P} \sum_{i=1}^{n} d_{i k L} x_{i k L} \leq R_{k L}, k=1, \ldots, p ; i=1, \ldots, n \\
x_{i k} \geq 0, k=1, \ldots, p ; i=1, \ldots, n
\end{array}\right.
\end{gathered}
$$

The entire model describes the process of decision-making. First, the top decision makers should give an allocation scheme that can affect the personnel. Headquarters aim to the goal that each branch fulfill tasks in a predetermined period and use the smallest amount of shared staff. Then, the lower use the personnel assigned to obtain the maximum economic value respectively, and return the results to the top decision makers. Top decision-makers adjust resource allocation scheme according to the requirement of target function. This process continues until the objective function of top decision-makers achieve optimal.

For this model, it has the following three major properties. $x^{*}{ }_{i k}$ indicates the number of optimum production of subordinate departments $R_{k L}^{*}$ refer to the optimal allocation scheme of headquarters.

Property1: For the results $\left(R^{*}{ }_{k L}, x^{*}{ }_{i k}\right)$,the induce necessary and sufficient condition is that, corresponding $R_{k L}$ can be found on any of $x_{\mathrm{ik}}$ to make the objective function meet this relationship $F\left(R_{k L}^{*}, x^{*}{ }_{i k}\right)>F\left(R_{k L}, x_{i k}\right)$.

Property2: If a set of results $\left(R_{k L}, x_{i k}\right)$ can establish the relationship like $F\left(R_{k L}, x_{i k}\right)>F\left(R_{k L}^{0}, x_{i k}^{0}\right)$, that is to say $\left(R_{k L}, x_{i k}\right)$ is inducible. If $F\left(R_{k L}, x_{i k}\right)<F\left(R_{k L}^{0}, x^{0}{ }_{i k}\right)$, it indicate that $\left(R_{k L}, x_{i k}\right)$ is not inducible.

Property3: If subordinate do not know the circumstances of any decision-making information of superiors, it must take min-max strategy. $\left(R^{0}{ }_{k L}, x^{0}{ }_{i k}\right)$ is the key to the problem below. Constraint conditions are like the equation (4).

$\max \omega_{k}=\sum_{i=1}^{n} c_{i k} x_{i k}, \quad k=1, \ldots, p ; i=1, \ldots, n$

$\min \omega_{k}=\sum_{i=1}^{n} c_{i k} x_{i k}, \quad k=1, \ldots, p ; i=1, \ldots, n$

Seen from the above properties, $F\left(R^{0}{ }_{k L}, x^{0}{ }_{i k}\right)$ is actually the last line of defense, but also the boundary of inducement. Thus solving the inducible two-tier can be divided into two 
stages. The first stage is to find lower levels of the last line of defense. The second stage is in the induced region to solve the main problem according to the forward master-slave strategy. Based on the analysis above, this paper proposes a new method which combines genetic algorithm and heuristic algorithm.

\section{ALGORITHMS IMPLEMENTATION}

Compared with other traditional search algorithm, genetic algorithm is characterized by avoiding locally optimal solution. In this paper, genetic algorithm [3,4] is used in the upper to deal with the constraints. At the same time, the model describes the decision-making process on the requirements of that top decision-makers give a shared staff allocation scheme in case, the lower decision model will be solved once. Because the underlying problem is an NP problem, it is possible to use genetic algorithms to solve. But two nested layers of genetic algorithm will greatly increase the computation. So, we use the heuristic algorithm [5] to solve the underlying problem. In this heuristic algorithm, priority rule [6] is used to solve the conflicts of multiple activities based on the competition of personnel [7].

\section{A. The Basic Elements of Specific Genetic Algorithm.}

- Generation and coding of initial population

This paper adopts real coding. Coding chromosome is the number of the working staff.

- Design fitness function

As the fitness function of genetic algorithm usually takes the maximum value, so here the fitness function takes the negative of the objective function.

$$
\begin{array}{r}
\text { Evaluation Function }=\max \left\{-\left(\varepsilon \sum_{L=1}^{m} \sum_{k=1}^{p} R_{k L}\right.\right. \\
\left.\left.+\sum_{k=1}^{p}\left(\max \left(\omega_{k}^{*}-\omega_{k}, 0\right)\right)\right)\right\}
\end{array}
$$

- Determine the choice of operator

In this paper, regular geometric ranking selection method is used. This selection method is to sort individual populations based on the value of its fitness function. The probability of each selected individual can be calculated by

equation like $p(i)=\frac{q}{1-(1-q)^{p}}(1-q)^{r-1}$. Sign $\mathrm{q}$ indicates the likelihood of the best individual which is selected, here take q 0.8. No. 1 represents the best. Here $r$ is the number of total individual, $\mathrm{p}$ represents the size of the population.

- Selection rules of crossover and mutation

Because we seek for integers, the mutation and crossover result rounding operation. Arithmetic crossover refers to the two chromosomes $X_{g}$ and $Y_{g}$ (g is the current algebra) produce two filial generations entirely by a linear combination of parents.

$$
\left\{\begin{array}{l}
X_{g}^{\prime}=\operatorname{int}\left(\mathrm{rX}_{\mathrm{g}}+(1-\mathrm{r}) Y_{g}\right) \\
Y_{g}^{\prime}=\operatorname{int}\left((1-r) X_{g}+r Y_{g}\right)
\end{array}\right.
$$

In the equation $(8), r=\mathrm{U}(0,1)$ means random number between 0 and 1 which obey uniform distribution. Because the parent generation meets the constraints, the offspring will not exceed the upper bound. If the chromosome $X_{g}=\left(x_{1}, \ldots, x_{m}\right)$ ( $\mathrm{g}$ is the current algebra) element $x_{i}$ is chosen for mutation. The obtained results are vector.

$$
x_{i}^{\prime}=\operatorname{int}\left\{\begin{array}{l}
x_{i}+\left(b_{i}-x_{i}\right) f(g) \text { if } \mathrm{r}_{1} \geq 0.5 \\
x_{i}+\left(x_{i}-a_{i}\right) f(g) \text { if } \mathrm{r}_{1}<0.5
\end{array}\right.
$$

In the equation (8), $a_{i}$ is the lower bound of variable $x_{i} ; b_{i}$ is the upper bound of variable $x_{i}$; $f(g)=\left(\mathrm{r}_{2}\left(1-\frac{g}{G_{\max }}\right)\right)^{b} ; r_{1}, r_{2}$ is random number which uniformly distributed between 0 and $1 ; \mathrm{g}$ is the current algebra; $G_{\max }$ is the maximum number of evolution; b represents a formal parameter.

- Select the termination criterion

Since the problem for solving distribution of the limited staff in multiple branches is not known in advance, so the termination criterion is solved through the given a maximum number of iterations.

\section{B. The Specific Calculation Steps.}

- Determine the basic elements of genetic algorithm.

- Value $\mathrm{k}=1$, then a number of shared personnel allocation scheme generates randomly. By judging from equation (7), all staff who meets shared personnel constraints will be regarded as individuals in the initial population.

- According to equation (7), the planned level of the objective function is transformed to the fitness function of genetic algorithm. For each branch, the way to solve the optimized problem is to call heuristic algorithm of subroutines. The result is then substituted into the planned level of the objective 
function, the corresponding fitness function value will be obtained.

- In accordance with the selected probability, individuals are selected. According to equation (8) and (9) respectively, the operation of crossover and mutation is performed, a new generation of individuals emerges.

- $\quad$ Take $\mathrm{k}=\mathrm{k}+1$, go to step3.

- When the maximum number of iterations is greater than the given $\mathrm{k}$, choose to terminate the genetic algorithm.

- Output the optimal solution.

\section{THE EXAMPLE}

In order to illustrate the application of the proposed method which is based on the inducible decision-making mechanism of the two-tier model is effective in solving the staff assignment, the method of allocating shared personnel at two branches.

The predetermined period of the projects is 54 days and 55 days respectively. The number of three kinds of shared staff distributed by corporation are 11,10,6 respectively. According to (1-4), using the algorithm proposed in this paper. Assumed that the duration of completed projects is inversely proportional to the amount of allocation. Therefore, the sub-project duration is equal to engineering effort divided by shared staff workload. With respect to the time limit for upper, the goal is to minimize the sharing staff. The target of the lower is to maximize value of production.

Design the interface, input and output are shown in Fig. 2. Input data and push the button of "run", then the results will be displayed. Click the "exit" button, exit the program interface.

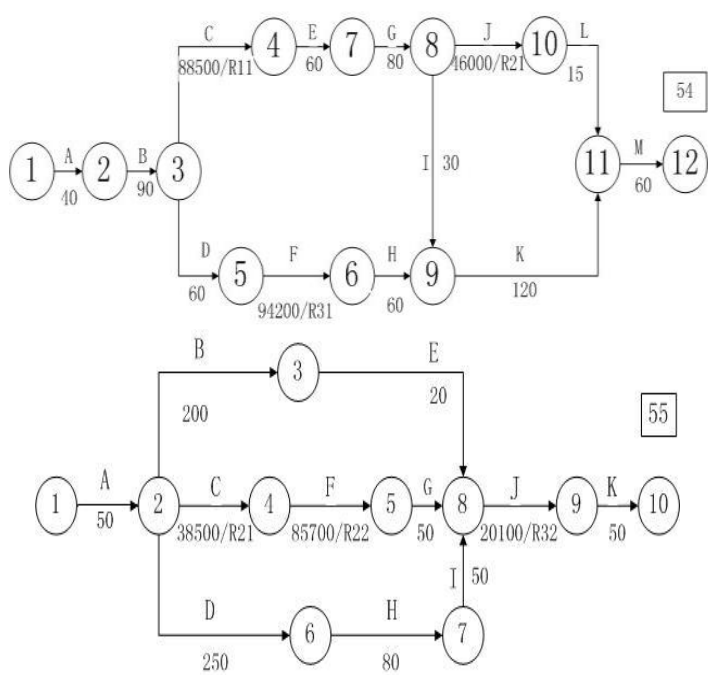

Figure 1. Two Projects

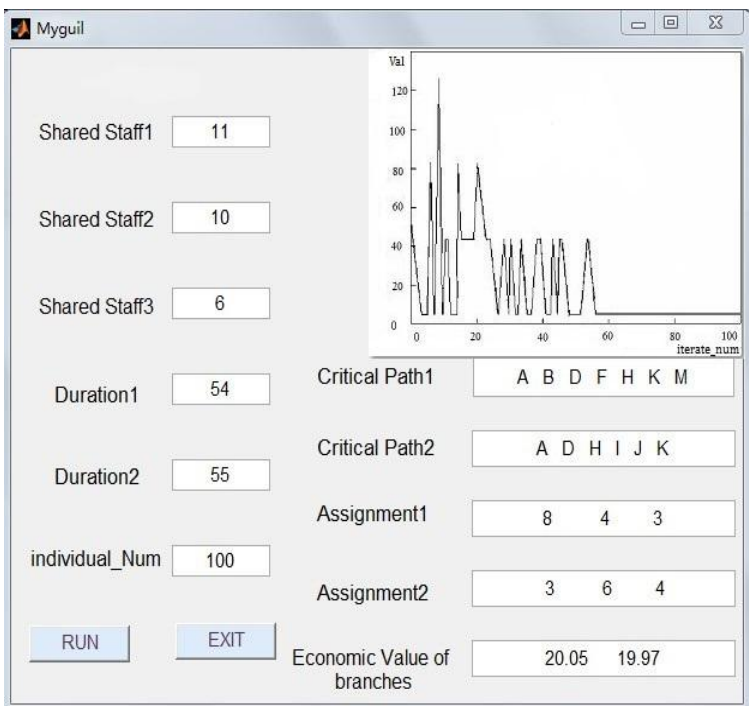

Figure 2. The Results

The input variables are three kinds of shared staff, a predetermined period of two branches and the number of individuals. Outputs include arrangement of production critical paths of two branches, shared staff assignments and economic value of branches.

We can see from Fig. 2 that genetic algorithm achieve to stability after 55 generation. Using genetic-heuristic algorithms obtain two branches of economic value 20.05 and 19.97.Compared to the initial data 19.26and19.13, as can be seen, the two branches of economic value has been improved to some extent. This shows that the algorithm is feasible.

\section{CONCLUSION}

Through combining the production-oriented enterprises in which limited staff allocation between multiple projects is widespread, this paper proposed the establishment of own resources unrestricted two-inducible decision model, and use genetic -- hybrid heuristic algorithm to solve the model. The calculation example shows that the use of the proposed method can basically get the global optimal solution, enable more people in more optimal allocation between projects and bring some economic benefits for enterprise.

\section{ACKNOWLEDGMENT}

I honestly express my appreciation to my mentor for his careful attention and meticulous guidance. I also would like to thank all schoolmates in the laboratory who give me selfless help, full support and enthusiastic encouragement. In addition, my thanks would go to my beloved friends who give me their help and time in listening to me and helping me work out my problems during the difficult course of the article. It is precisely because of your help and support, I can overcome difficulties and doubts, until the successful completion of this paper. 
This work was supported by Natural Science Foundation

Project of NSFC under the grant NO.60872157 and

NO.61271007.

\section{REFERENCES}

[1] Ma Hong. Research for resource allocation problem of two decision models.2004.

[2] Karlof J.K.,Wang. Bilevel programming applied to the flow shop scheduling problem[J]. Computers \& Operations Research,1996,Vo1.23,No.5: 443 451.

[3] Y. Muroi, T. Nishi, M. Inuiguchi. Improvement of column generation method for railway crew scheduling problems. IEEE Transactions on Electronics, Information and Systems, 2010, 130(2):275-283.

[4] Sou Sen Leu,Chung Huei Yang.GA based multi criteria optimal model for construction scheduling[J].Journal of Construction Engineering and Management,1999,125(6):420-42

[5] Kolisch R. Project scheduling under resource constraints-Efficient heuristics for several problem classes. Physica,Heidelberg, 1995.

[6] Kelley J.E., Jr. The critical path method: Resource planning and scheduling. Ch. 21 in Industrial Scheduling, J.F. Muth and G.L. Thompson (eds.) ,Prentice-Hall, Englewood Cliffs, NJ,1963,p.347-365.

[7] LEE J-K, KIM Y-D. Search heuristics for resource constrained project scheduling. Journal of The Operational Research Society, 1996, Vol.47, No.5, p.678-689. 